\title{
Probing the Planar Tetra-, Penta-, and Hexa-coordinate Carbon in \\ Carbon-Boron Mixed Clusters
}

\author{
Yong Pei and Xiao Cheng Zeng* \\ Department of Chemistry and Nebraska Center for Materials and Nanoscience, \\ University of Nebraska-Lincoln, Lincoln, Nebraska 68588 \\ xczeng@phase2.unl.edu
}

\section{Contents}

I. Full citation of reference 42

II. Top three low-lying isomers of clusters $\mathrm{C}_{m=1 \sim 4} \mathrm{~B}_{n=4 \sim 8}$ and their anions.

III. Geometrical data for structures given in Figure 4.

IV. Infrared spectrum of the $\mathrm{CB}_{4}$. 


\section{Full citation of reference 42}

Gaussian 03, Revision C.02, Frisch, M. J.; Trucks, G. W.; Schlegel, H. B.; Scuseria, G. E.; Robb, M. A.; Cheeseman, J. R.; Montgomery, Jr., J. A.; Vreven, T.; Kudin, K. N.; Burant, J. C.; Millam, J. M.; Iyengar, S. S.; Tomasi, J.; Barone, V.; Mennucci, B.; Cossi, M.; Scalmani, G.; Rega, N.; Petersson, G. A.; Nakatsuji, H.; Hada, M.; Ehara, M.; Toyota, K.; Fukuda, R.; Hasegawa, J.; Ishida, M.; Nakajima, T.; Honda, Y.; Kitao, O.; Nakai, H.; Klene, M.; Li, X.; Knox, J. E.; Hratchian, H. P.; Cross, J. B.; Bakken, V.; Adamo, C.; Jaramillo, J.; Gomperts, R.; Stratmann, R. E.; Yazyev, O.; Austin, A. J.; Cammi, R.; Pomelli, C.; Ochterski, J. W.; Ayala, P. Y.; Morokuma, K.; Voth, G. A.; Salvador, P.; Dannenberg, J. J.; Zakrzewski, V. G.; Dapprich, S.; Daniels, A. D.; Strain, M. C.; Farkas, O.; Malick, D. K.; Rabuck, A. D.; Raghavachari, K.; Foresman, J. B.; Ortiz, J. V.; Cui, Q.; Baboul, A. G.; Clifford, S.; Cioslowski, J.; Stefanov, B. B.; Liu, G.; Liashenko, A.; Piskorz, P.; Komaromi, I.; Martin, R. L.; Fox, D. J.; Keith, T.; Al-Laham, M. A.; Peng, C. Y.; Nanayakkara, A.; Challacombe, M.; Gill, P. M. W.; Johnson, B.; Chen, W.; Wong, M. W.; Gonzalez, C.; and Pople, J. A.; Gaussian, Inc., Wallingford CT, 2004. 
II. Top three low-lying isomers of clusters $C_{m=1 \sim 4} B_{n=4 \sim 8}$ and their anions. (The absolute energy of each isomer is given underneath the isomer structure, in unit of Hartree. The value in parentheses represents relative energy, in unit of $\mathbf{k c a l} / \mathbf{m o l})$.

$\mathrm{CB}_{4}$

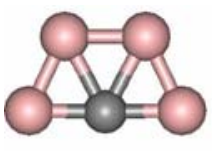

$\left(\mathrm{C}_{2 v},{ }^{1} \mathrm{~A}_{1}\right)$

B3LYP/6-311+G(d,p)

MP2/cc-pVTZ

CCSD(T)/cc-pVTZ//

MP2/cc-pVTZ

$-136.8888(\mathbf{0 . 0 0})$

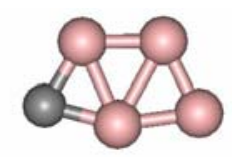

$\left(\mathrm{C}_{1},{ }^{1} \mathrm{~A}\right)$

$-137.2430(-7.04)$

$-136.8236(6.08)$

$-136.8861(\mathbf{1 . 6 7})$

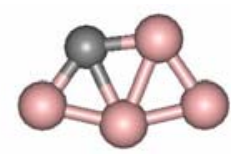

$\left(\mathrm{C}_{1},{ }^{3} \mathrm{~A}\right)$

$-137.2461(-8.97)$

$-136.81993(\mathbf{8 . 3 8})$

$-136.8811(\mathbf{4 . 8 4})$

\section{$\mathrm{CB}_{4}{ }^{-}$}

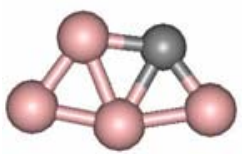

$\left(\mathrm{C}_{1},{ }^{2} \mathrm{~A}\right)$

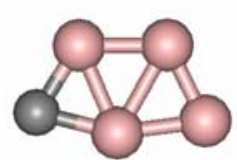

$\left(\mathrm{C}_{1},{ }^{2} \mathrm{~A}\right)$

$\left(\mathrm{C}_{1},{ }^{2} \mathrm{~A}\right)$

$\begin{array}{llll}\text { B3LYP } / 6-311+G(d, p) & -137.3266(\mathbf{0 . 0 0}) & -137.3395(\mathbf{- 8 . 0 8}) & -137.3120(\mathbf{9 . 1 8}) \\ & & & \\ \text { CCSD }(T) / 6-311+G(d, p) & -136.9212(\mathbf{0 . 0 0}) & -136.9101(\mathbf{6 . 9 9}) & -136.8836(\mathbf{2 3 . 5 8})\end{array}$




\section{$\mathrm{CB}_{5}$}

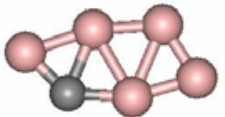

$$
\left(\mathrm{C}_{s},{ }^{2} \mathrm{~A}^{\prime}\right)
$$

B3LYP/6-311+G(d,p) $\quad-162.0948(\mathbf{0 . 0 0})$

CCSD(T)/cc-pVTZ $\quad-161.6623(\mathbf{0 . 0 0})$

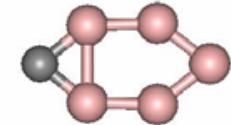

$\left(\mathrm{C}_{2 v},{ }^{2} \mathrm{~A}_{1}\right)$

$-162.1071(-7.39)$

$-161.6619(\mathbf{0 . 2 3})$

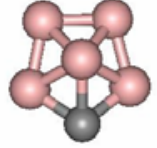

$\left(\mathrm{C}_{s},{ }^{2} \mathrm{~A}^{\prime}\right)$ $-162.0601(\mathbf{2 1 . 7 9 )}$ $-161.6343(\mathbf{1 7 . 5 2})$

\section{$\mathrm{CB}_{5}{ }^{-}$}

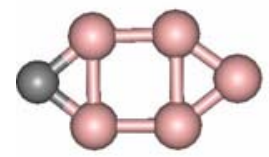

$\left(\mathrm{C}_{2 v},{ }^{1} \mathrm{~A}_{1}\right)$

B3LYP/6-311+G(d,p) $\quad-162.2221(0.00)$

$\operatorname{CCSD}(\mathrm{T}) / 6-311+\mathrm{G}(\mathrm{d}, \mathrm{p}) \quad-161.7126(\mathbf{0 . 0 0})$

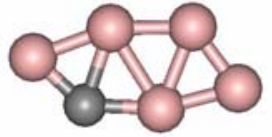

$\left(\mathrm{C}_{s},{ }^{1} \mathrm{~A}^{\prime}\right)$

$-162.1986(\mathbf{1 4 . 7 7})$

$-162.1937(\mathbf{1 7 . 8 2})$

$-161.6964(\mathbf{1 0 . 1 8})$
$\left(\mathrm{C}_{s},{ }^{3} \mathrm{~A}^{\prime}\right)$

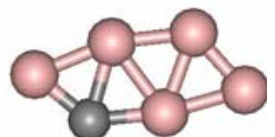

$-161.6791(\mathbf{2 1 . 0 2})$ 


\section{$\mathrm{CB}_{6}$}

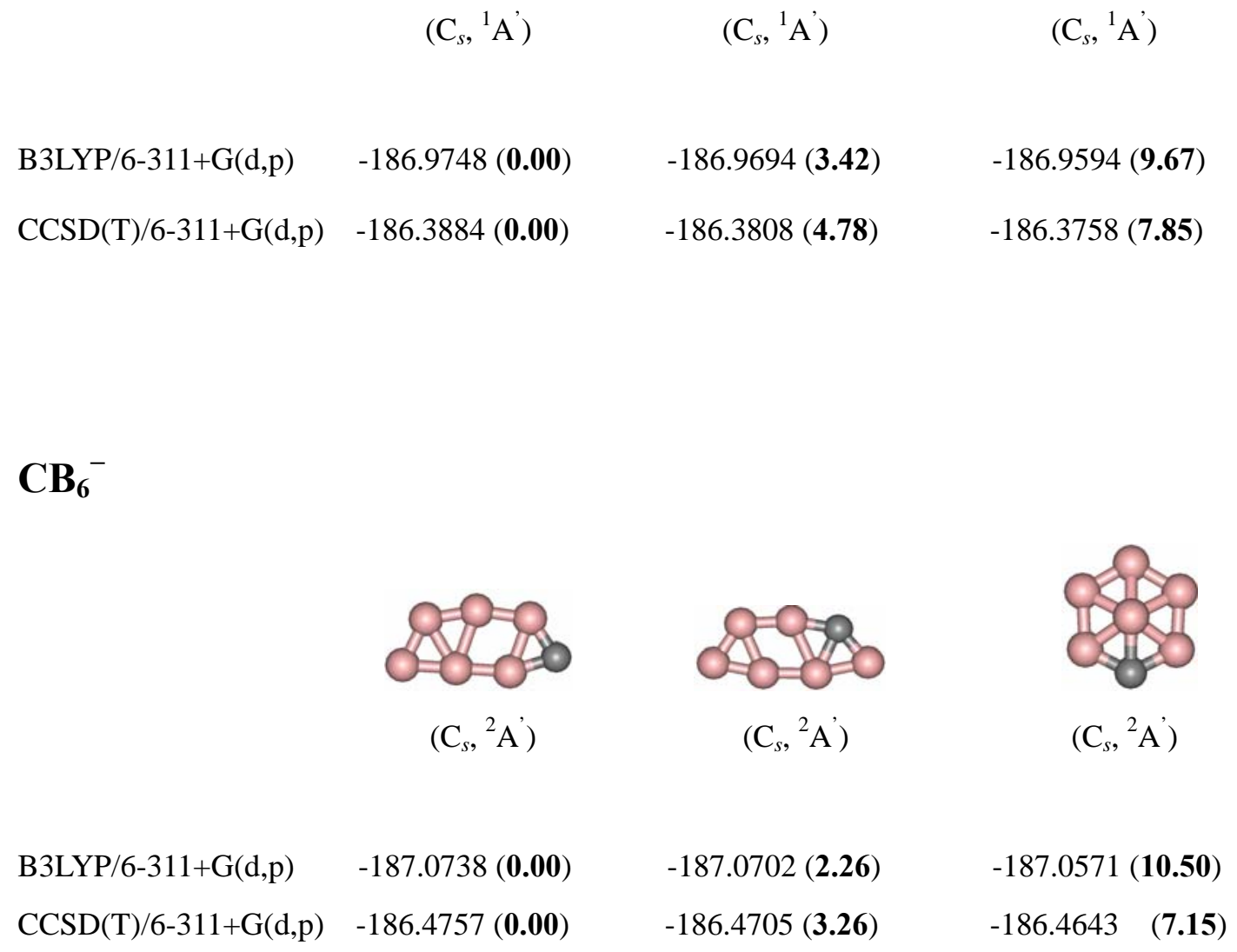

$\left(\mathrm{C}_{s},{ }^{1} \mathrm{~A}^{\prime}\right)$

B3LYP/6-311+G(d,p) $\quad-186.9748(\mathbf{0 . 0 0})$

$\operatorname{CCSD}(\mathrm{T}) / 6-311+\mathrm{G}(\mathrm{d}, \mathrm{p}) \quad-186.3884(\mathbf{0 . 0 0})$

$\mathrm{CB}_{6}{ }^{-}$

$\left(\mathrm{C}_{s},{ }^{2} \mathrm{~A}^{\prime}\right)$

B3LYP/6-311+G(d,p) $\quad-187.0738(\mathbf{0 . 0 0})$

$-187.0702(\mathbf{2 . 2 6})$

-186.4705 (3.26)

$$
\left(\mathrm{C}_{s},{ }^{1} \mathrm{~A}^{\prime}\right)
$$

$-186.9694(\mathbf{3 . 4 2})$

$-186.9594(\mathbf{9 . 6 7})$

$-186.3758(7.85)$

$\left(\mathrm{C}_{s},{ }^{2} \mathrm{~A}\right)$

$\left(\mathrm{C}_{s},{ }^{2} \mathrm{~A}^{\prime}\right)$

CCSD(T)/6-311+G(d,p) -186.4757 (0.00)

$-186.4643 \quad(7.15)$ 


\section{$\mathrm{CB}_{7}$}

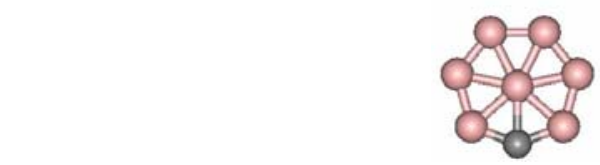

$\left(\mathrm{C}_{2 v},{ }^{2} \mathrm{~A}_{1}\right)$

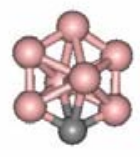

$\left(\mathrm{C}_{2 v},{ }^{2} \mathrm{~A}_{1}\right)$

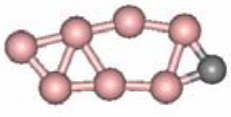

$\left(\mathrm{C}_{s},{ }^{2} \mathrm{~A}^{\prime}\right)$

B3LYP/6-311+G(d,p) $\quad-211.8724(\mathbf{0 . 0 0}) \quad-211.8092(\mathbf{3 9 . 6 8}) \quad-211.8127(\mathbf{3 7 . 4 7})$

$\operatorname{CCSD}(\mathrm{T}) / 6-311+\mathrm{G}(\mathrm{d}, \mathrm{p}) \quad-211.3067(\mathbf{0 . 0 0}) \quad-211.2636(\mathbf{2 7 . 0 9}) \quad-211.2404$ (41.66)

\section{$\mathrm{CB}_{7}^{-}$}

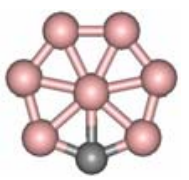

$\left(\mathrm{C}_{2 v},{ }^{1} \mathrm{~A}_{1}\right)$

B3LYP/6-311+G(d,p) $\quad-211.9775(\mathbf{0 . 0 0})$

$\operatorname{CCSD}(\mathrm{T}) / 6-311+\mathrm{G}(\mathrm{d}, \mathrm{p}) \quad-186.3884(\mathbf{0 . 0 0})$

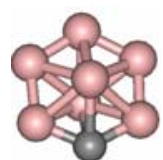

$\left(\mathrm{C}_{2 v},{ }^{1} \mathrm{~A}_{1}\right)$

-211.8927 (53.19)

-186.3808 (35.58)

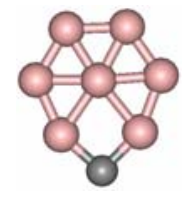

$\left(\mathrm{C}_{s},{ }^{3} \mathrm{~A}^{\prime}\right)$

-211.9209 (35.49)

-186.3758 (38.96) 


\section{$\mathbf{C B}_{8}$}

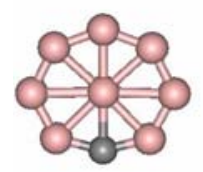

$\left(\mathrm{C}_{2 v},{ }^{1} \mathrm{~A}_{1}\right)$

B3LYP/6-311+G(d,p) $\quad-236.7082(\mathbf{0 . 0 0})$

CCSD(T)/6-311+G(d,p) $-235.9520(\mathbf{0 . 0 0})$

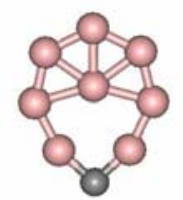

$\left(\mathrm{C}_{2 v},{ }^{3} \mathrm{~A}_{2}\right)$

$-236.6869(\mathbf{1 3 . 4 3})$

$-235.9253(\mathbf{1 6 . 7 5 )}$

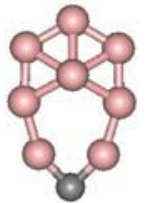

$\left(\mathrm{C}_{s},{ }^{1} \mathrm{~A}^{\prime}\right)$

\section{$\mathrm{CB}_{8}{ }^{-}$}

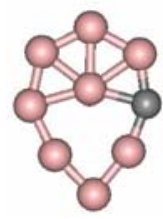

$\left(\mathrm{C}_{s},{ }^{2} \mathrm{~A}^{\prime}\right)$

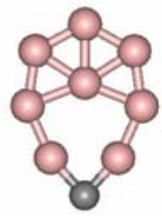

$\left(\mathrm{C}_{2 v},{ }^{2} \mathrm{~A}_{2}\right)$

B3LYP/6-311+G(d,p) $\quad-236.8057(\mathbf{0 . 0 0}) \quad-236.8072(\mathbf{- 0 . 9 3}) \quad-236.7940(\mathbf{7 . 3 4})$

$\begin{array}{llll}\operatorname{CCSD}(\mathrm{T}) / 6-311+G(\mathrm{~d}, \mathrm{p}) & -235.9520(\mathbf{0 . 0 0}) & -235.9253(\mathbf{0 . 6 4}) & -235.9229(\mathbf{6 . 5 2})\end{array}$

$\begin{array}{lll}\mathrm{CCSD}(\mathrm{T}) / \mathrm{cc}-\mathrm{pVTZ} & -236.1685(\mathbf{0 . 0 0}) & -236.1664(\mathbf{1 . 2 6})\end{array}$

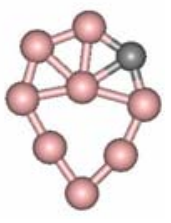

$\left(\mathrm{C}_{s},{ }^{2} \mathrm{~A}^{\prime}\right)$ 


\section{$\mathrm{C}_{2} \mathrm{~B}_{4}$}

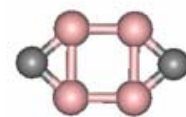

$$
\left(\mathrm{D}_{2 h},{ }^{1} \mathrm{~A}_{\mathrm{g}}\right)
$$

B3LYP/6-311+G(d,p)

$\operatorname{CCSD}(\mathrm{T}) / 6-311+\mathrm{G}(\mathrm{d}, \mathrm{p})$

$-175.4165(\mathbf{0 . 0 0})$

$-174.8905(\mathbf{0 . 0 0})$

\section{$\mathrm{C}_{2} \mathrm{~B}_{4}{ }^{-}$}

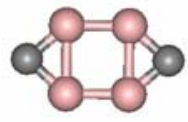

$$
\left(D_{2 h},{ }^{2} A_{g}\right)
$$

B3LYP/6-311+G(d,p)

$-175.4895(\mathbf{0 . 0 0})$

$\operatorname{CCSD}(\mathrm{T}) / 6-311+\mathrm{G}(\mathrm{d}, \mathrm{p}) \quad-174.9490(\mathbf{0 . 0 0})$

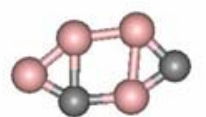

$\left(\mathrm{C}_{s},{ }^{1} \mathrm{~A}^{\prime}\right)$

$-175.3926(\mathbf{1 4 . 9 5})$

$-174.8683(\mathbf{1 3 . 9 7})$

$-175.3792(\mathbf{2 3 . 4 1})$

$-174.8601(\mathbf{1 9 . 0 8})$

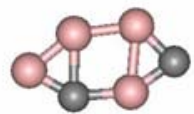

$\left(\mathrm{C}_{s},{ }^{2} \mathrm{~A}^{\prime}\right)$

$-175.4792(6.48)$ $-174.9406(\mathbf{5 . 2 3})$

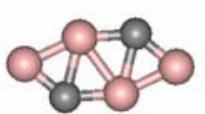

$\left(\mathrm{C}_{2 h},{ }^{1} \mathrm{~A}_{\mathrm{g}}\right)$

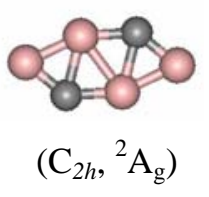

$-175.4742(9.59)$

-174.9355 (8.44) 


\section{$\mathbf{C}_{2} \mathbf{B}_{5}$}

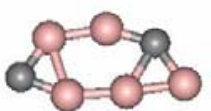

$\left(\mathrm{C}_{s},{ }^{2} \mathrm{~A}^{\prime}\right)$

B3LYP/6-311+G(d,p) $\quad-200.2503(\mathbf{0 . 0 0})$

$\operatorname{CCSD}(T) / 6-311+G(d, p)$

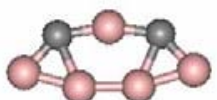

$\left(\mathrm{C}_{2 v},{ }^{2} \mathrm{~A}_{1}\right)$

$-200.2431(\mathbf{4 . 5 3})$

-199.6247 (5.91)

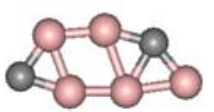

B3LYP/6-311+G(d,p) $\quad-200.3386(\mathbf{0 . 0 0})$

$\operatorname{CCSD}(\mathrm{T}) / 6-311+\mathrm{G}(\mathrm{d}, \mathrm{p})$

$\operatorname{CCSD}(\mathrm{T}) / \mathrm{cc}-\mathrm{pVTZ}$

$$
\left(\mathrm{C}_{2 v},{ }^{1} \mathrm{~A}_{1}\right)
$$

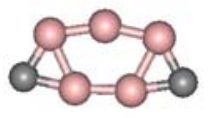

-199.7191 (0.00)

$-199.8203(\mathbf{0 . 0 0})$
$\left(\mathrm{C}_{s},{ }^{1} \mathrm{~A}^{\prime}\right)$

$-200.3360(\mathbf{1 . 5 9})$

$-199.7185(\mathbf{0 . 3 8})$

$-199.8192(\mathbf{0 . 6 8})$

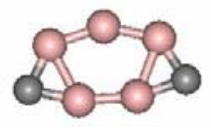

$\left(\mathrm{C}_{2 v},{ }^{2} \mathrm{~A}_{1}\right)$

$-200.2374(\mathbf{8 . 1 4})$

$-199.6195(\mathbf{9 . 1 2})$

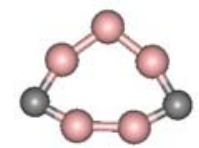

$\left(\mathrm{C}_{2 v},{ }^{3} \mathrm{~A}_{1}\right)$

$-200.3310(4.77)$

$-199.7115(4.77)$

$-199.8113(\mathbf{5 . 6 4 )}$ 


\section{$\mathrm{C}_{2} \mathrm{~B}_{6}$}

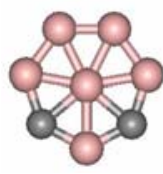

$\left(\mathrm{C}_{2 v},{ }^{1} \mathrm{~A}_{1}\right)$

B3LYP/6-311+G(d,p)

$\operatorname{CCSD}(T) / 6-311+G(d, p)$

CCSD(T)/cc-pVTZ

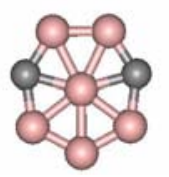

$\left(\mathrm{C}_{2 v},{ }^{1} \mathrm{~A}_{1}\right)$
$\left(\mathrm{C}_{2 v},{ }^{1} \mathrm{~A}_{1}\right)$

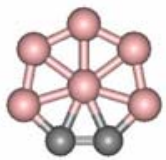

\section{$\mathrm{C}_{2} \mathrm{~B}_{6}^{-}$}

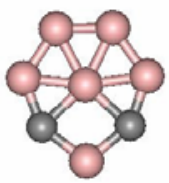

$\left(\mathrm{C}_{2 v},{ }^{2} \mathrm{~A}_{1}\right)$

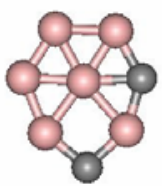

$\left(\mathrm{C}_{s},{ }^{2} \mathrm{~A}^{\prime}\right)$

$-225.1899(\mathbf{3 . 1 4})$

$-224.4926(6.09)$

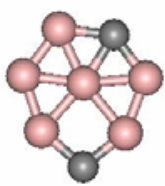

$\left(\mathrm{C}_{s},{ }^{2} \mathrm{~A}^{\prime}\right)$

B3LYP/6-311+G(d,p) $\quad-225.1949(\mathbf{0 . 0 0})$

$-224.5023(\mathbf{0 . 0 0})$ 


\section{$\mathbf{C}_{2} \mathbf{B}_{7}$}
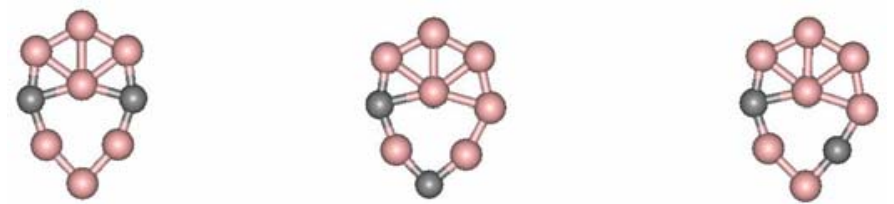

$\left(\mathrm{C}_{2 v},{ }^{2} \mathrm{~A}_{1}\right)$

$\left(\mathrm{C}_{\mathrm{s}},{ }^{2} \mathrm{~A}^{\prime}\right)$

$\left(\mathrm{C}_{\mathrm{s}},{ }^{2} \mathrm{~A}^{\prime}\right)$

B3LYP/6-311+G(d,p)

-249.9737 (0.00)

$-249.9730(\mathbf{0 . 4 8})$

$-249.9660(\mathbf{4 . 8 4})$

CCSD(T)/6-311+G(d,p)

-249.1957 (0.00)

-249.1939 (1.12)

-249.1876 (5.08)

CCSD(T)/cc-pVTZ

$-249.3242(\mathbf{0 . 0 0})$

-249.3219 (1.44)

\section{$\mathbf{C}_{2} \mathbf{B}_{7}^{-}$}

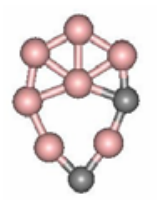

$\left(\mathrm{C}_{s},{ }^{1} \mathrm{~A}^{\prime}\right)$

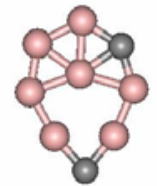

$\left(\mathrm{C}_{s},{ }^{1} \mathrm{~A}^{\prime}\right)$

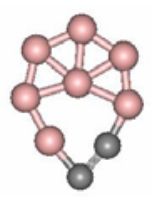

$\left(\mathrm{C}_{s},{ }^{1} \mathrm{~A}^{\prime}\right)$

B3LYP/6-311+G(d,p) $\quad-250.0926(\mathbf{0 . 0 0})$

$-250.0798(\mathbf{8 . 0 6})$

$-250.0659(\mathbf{1 6 . 7 7})$

$\operatorname{CCSD}(\mathrm{T}) / 6-311+\mathrm{G}(\mathrm{d}, \mathrm{p}) \quad-249.3139(\mathbf{0 . 0 0})$

$-249.3014(7.87)$

-249.2880 (16.20) 


\section{$\mathrm{C}_{2} \mathrm{~B}_{8}$}

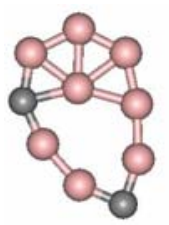

$\left(\mathrm{C}_{\mathrm{s}},{ }^{1} \mathrm{~A}^{\prime}\right)$

B3LYP/6-311+G(d,p)

CCSD(T)/6-311+G(d,p)

$-274.8428(\mathbf{0 . 0 0})$

$-273.9840(\mathbf{0 . 0 0})$

\section{$\mathrm{C}_{2} \mathrm{~B}_{8}{ }^{-}$}

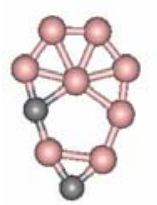

B3LYP/6-311+G(d,p)

$\operatorname{CCSD}(\mathrm{T}) / 6-311+\mathrm{G}(\mathrm{d}, \mathrm{p})$

CCSD(T)/cc-pVTZ
$\left(\mathrm{C}_{s},{ }^{2} \mathrm{~A}^{\prime}\right)$

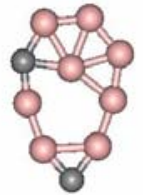

$-274.9292(\mathbf{0 . 0 0})$

$-274.0668(\mathbf{0 . 0 0})$

-274.2097 (0.00)

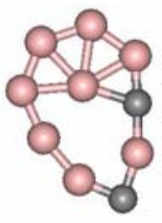

$\left(\mathrm{C}_{s},{ }^{1} \mathrm{~A}^{\prime}\right)$

$-274.8351(\mathbf{4 . 8 4})$

-273.9759 (5.05)

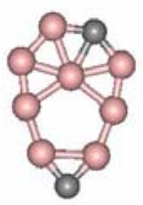

$\left(\mathrm{C}_{s},{ }^{2} \mathrm{~A}^{\prime}\right)$

-274.9262 (1.87)

-274.0643 (1.57)

$\left(\mathrm{C}_{s},{ }^{2} \mathrm{~A}^{\prime}\right)$

$-274.9174(7.39)$

-274.0562 (6.68)
-274.8256 (10.84)

-273.9681 (9.95)

-274.2067 (1.88) 


\section{$\mathrm{C}_{3} \mathrm{~B}_{4}$}

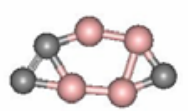

$\left(\mathrm{C}_{s},{ }^{1} \mathrm{~A}^{\prime}\right)$

B3LYP/6-311+G(d,p) -213.4892 (0.00)

$\operatorname{CCSD}(\mathrm{T}) / 6-311+\mathrm{G}(\mathrm{d}, \mathrm{p}) \quad-212.8548(\mathbf{0 . 0 0})$

\section{$\mathrm{C}_{3} \mathrm{~B}_{4}{ }^{-}$}

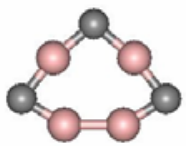

$$
\left(\mathrm{C}_{2 v},{ }^{2} \mathrm{~A}_{1}\right)
$$

B3LYP/6-311+G(d,p) $\quad-213.6006(\mathbf{0 . 0 0})$

$\operatorname{CCSD}(\mathrm{T}) / 6-311+G(\mathrm{~d}, \mathrm{p}) \quad-212.9578(\mathbf{0 . 0 0})$

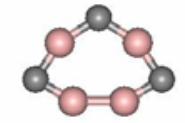

$\left(\mathrm{C}_{2 v},{ }^{3} \mathrm{~B}_{1}\right)$

$\left(\mathrm{C}_{s},{ }^{1} \mathrm{~A}^{\prime}\right)$

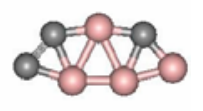

$-213.4841(\mathbf{3 . 2 0}) \quad-213.4758(\mathbf{8 . 4 4})$

$-212.8481(\mathbf{4 . 2 0}) \quad-212.8462(\mathbf{5 . 4 4})$

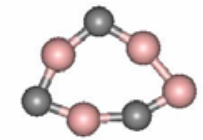

$\left(\mathrm{C}_{s},{ }^{2} \mathrm{~A}^{\prime}\right)$

$-213.5783(\mathbf{1 4 . 0 0}) \quad-213.5773(\mathbf{1 4 . 6 3})$

$-212.9356(\mathbf{1 3 . 9 1}) \quad-212.9334(\mathbf{1 5 . 3 1})$ 


\section{$\mathbf{C}_{3} \mathbf{B}_{5}$}

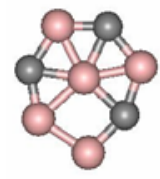

$\left(\mathrm{C}_{s},{ }^{2} \mathrm{~A}^{\prime}\right)$

B3LYP/6-311+G(d,p)

$\operatorname{CCSD}(\mathrm{T}) / 6-311+\mathrm{G}(\mathrm{d}, \mathrm{p})$

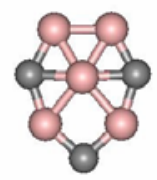

$\left(\mathrm{C}_{s},{ }^{2} \mathrm{~A}^{\prime}\right)$

$-238.3477(\mathbf{0 . 0 0})$

$-237.6369(\mathbf{0 . 0 0})$

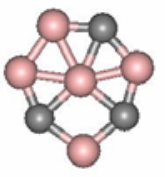

$\left(\mathrm{C}_{s},{ }^{2} \mathrm{~A}^{\prime}\right)$

$-238.3418(3.70)$

$-237.6336(\mathbf{2 . 0 8})$

$-237.6295(\mathbf{4 . 6 8})$

\section{$\mathrm{C}_{3} \mathrm{~B}_{5}^{-}$}

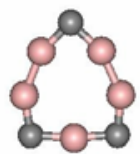

$\left(\mathrm{C}_{2 v},{ }^{1} \mathrm{~A}_{1}\right)$

B3LYP/6-311+G(d,p) $\quad-238.4899(\mathbf{0 . 0 0})$

$\operatorname{CCSD}(\mathrm{T}) / 6-311+\mathrm{G}(\mathrm{d}, \mathrm{p}) \quad-237.7670(\mathbf{0 . 0 0})$

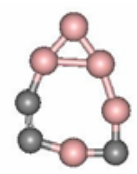

$\left(\mathrm{C}_{s},{ }^{1} \mathrm{~A}^{\prime}\right)$

-238.4625 (17.19)

$-238.4640(\mathbf{1 6 . 2 2})$

$-237.7440(\mathbf{1 4 . 4 5})$
$\left(\mathrm{C}_{s},{ }^{1} \mathrm{~A}^{\prime}\right)$

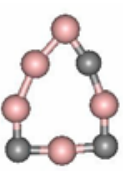

$-237.7435(\mathbf{1 4 . 7 8})$ 


\section{$\mathrm{C}_{3} \mathrm{~B}_{6}$}

\begin{tabular}{|c|c|c|c|}
\hline & $\left(\mathrm{C}_{2 v},{ }^{1} \mathrm{~A}_{1}\right)$ & $\left(\mathrm{C}_{2 v},{ }^{1} \mathrm{~A}_{1}\right)$ & $\left(\mathrm{C}_{s}, \mathrm{~A}^{\prime}\right)$ \\
\hline B3LYP/6-311+G(d,p) & $-263.2507(\mathbf{0 . 0 0})$ & $-263.2465(\mathbf{2 . 6 1})$ & $-263.2374(\mathbf{8 . 3 1})$ \\
\hline $\operatorname{CCSD}(\mathrm{T}) / 6-311+\mathrm{G}(\mathrm{d}, \mathrm{p})$ & $-262.4537(\mathbf{0 . 0 0})$ & $-262.4496(\mathbf{1 4 . 4 5})$ & $-262.4409(\mathbf{1 4 . 7 8})$ \\
\hline
\end{tabular}

\section{$\mathrm{C}_{3} \mathrm{~B}_{6}{ }^{-}$}
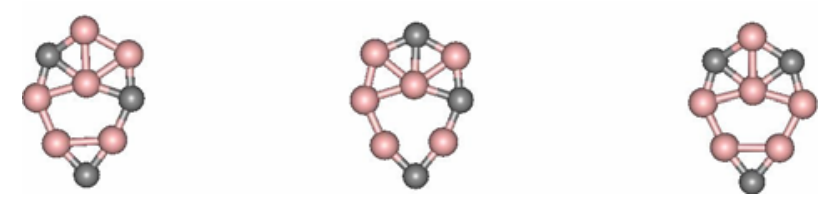

$\left(\mathrm{C}_{1},{ }^{2} \mathrm{~A}_{1}\right)$

$\left(\mathrm{C}_{s},{ }^{2} \mathrm{~A}^{\prime}\right)$

$\left(\mathrm{C}_{s},{ }^{2} \mathrm{~A}^{\prime}\right)$

B3LYP/6-311+G(d,p)

$-263.3042(\mathbf{0 . 0 0})$

$-263.3057(-\mathbf{0 . 9 0})$

$-263.2932(6.91)$

CCSD(T)/6-311+G(d,p)

$-262.5049(\mathbf{0 . 0 0})$

$-262.5046(\mathbf{0 . 1 9})$

$-262.4949(\mathbf{6 . 2 4})$

CCSD(T)/cc-pVTZ

$-262.6440(\mathbf{0 . 0 0})$

$-262.6426(\mathbf{0 . 9 0})$ 


\section{$\mathbf{C}_{3} \mathbf{B}_{7}$}

$\begin{array}{cccc} & & & \\ & \left(\mathrm{C}_{2 v}{ }^{2} \mathrm{~A}_{1}\right) & \left(\mathrm{C}_{\mathrm{s}},{ }^{2} \mathrm{~A}^{\prime}\right) & \\ \mathrm{B} 3 \mathrm{LYP} / 6-311+\mathrm{G}(\mathrm{d}, \mathrm{p}) & -288.0891(\mathbf{0 . 0 0}) & -288.0740(\mathbf{9 . 4 8}) & -288.0490(\mathbf{2 5 . 1 6}) \\ \mathrm{CCSD}(\mathrm{T}) / 6-311+\mathrm{G}(\mathrm{d}, \mathrm{p}) & -287.2090(\mathbf{0 . 0 0}) & -287.1930(\mathbf{1 0 . 0 4}) & -287.1689(\mathbf{2 5 . 1 6})\end{array}$

\section{$\mathbf{C}_{3} \mathbf{B}_{7}^{-}$}

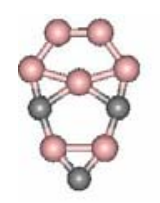

$$
\left(\mathrm{C}_{2 v},{ }^{1} \mathrm{~A}_{1}\right)
$$

B3LYP/6-311+G(d,p) $\quad-288.1816$ (0.00)

$\operatorname{CCSD}(\mathrm{T}) / 6-311+\mathrm{G}(\mathrm{d}, \mathrm{p}) \quad-287.3029(\mathbf{0 . 0 0})$

$\operatorname{CCSD}(\mathrm{T}) / \mathrm{cc}-\mathrm{pVTZ}$

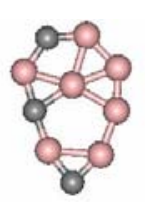

$\left(\mathrm{C}_{\mathrm{s}},{ }^{1} \mathrm{~A}^{\prime}\right)$

$-288.1814(\mathbf{0 . 1 3})$

$-287.3030(-\mathbf{0 . 0 7})$

$-287.4552(\mathbf{0 . 1 7})$

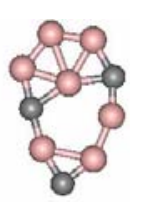

$\left(\mathrm{C}_{s},{ }^{1} \mathrm{~A}^{\prime}\right)$

-288.1765 (3.16)

$-287.2971(\mathbf{3 . 6 4})$ 


\section{$\mathrm{C}_{3} \mathrm{~B}_{8}$}

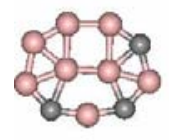

$\left(\mathrm{C}_{\mathrm{s}},{ }^{1} \mathrm{~A}^{\prime}\right)$

B3LYP/6-311+G(d,p) $\quad-312.9224(\mathbf{0 . 0 0})$

CCSD(T)/6-311+G(d,p) -311.9665 (0.00)

\section{$\mathrm{C}_{3} \mathrm{~B}_{8}{ }^{-}$}

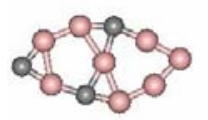

$$
\left(\mathrm{C}_{s},{ }^{2} \mathrm{~A}^{\prime}\right)
$$

B3LYP/6-311+G(d,p) $\quad-312.9224(\mathbf{0 . 0 0})$

CCSD(T)/6-311+G(d,p) -311.9665 (0.00)
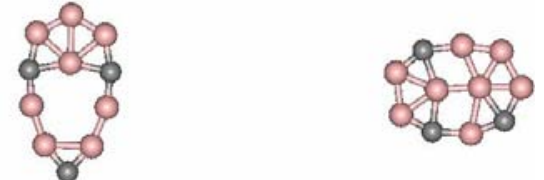

$\left(\mathrm{C}_{2 v},{ }^{1} \mathrm{~A}_{1}\right)$

$\left(\mathrm{C}_{s},{ }^{1} \mathrm{~A}^{\prime}\right)$

$-312.9244(-\mathbf{1 . 2 5}) \quad-312.9154(\mathbf{4 . 3 7})$

$-311.9626(\mathbf{2 . 4 2}) \quad-311.9626(\mathbf{2 . 4 5})$

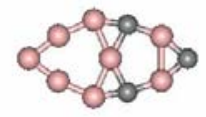

$\left(\mathrm{C}_{s},{ }^{2} \mathrm{~A}^{\prime}\right)$

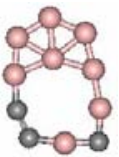

$\left(\mathrm{C}_{s},{ }^{2} \mathrm{~A}^{\prime}\right)$

$-312.9244(\mathbf{2 . 4 0})$

-312.9154 (4.60)

-311.9626 (2.80)

-311.9626 (9.91) 


\section{$\mathrm{C}_{4} \mathrm{~B}_{4}$}

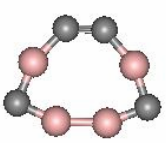

$\left(\mathrm{C}_{2 v},{ }^{1} \mathrm{~A}_{1}\right)$

B3LYP/6-311+G(d,p)

$\operatorname{CCSD}(\mathrm{T}) / 6-311+\mathrm{G}(\mathrm{d}, \mathrm{p})$

$-251.6482(\mathbf{0 . 0 0})$

$-250.9130(\mathbf{0 . 0 0})$

\section{$\mathrm{C}_{4} \mathrm{~B}_{4}{ }^{-}$}

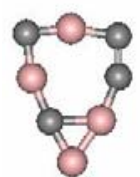

$\left(\mathrm{C}_{s},{ }^{2} \mathrm{~A}^{\prime}\right)$

B3LYP/6-311+G(d,p)

$-251.7192(\mathbf{0 . 0 0})$

$\operatorname{CCSD}(\mathrm{T}) / 6-311+\mathrm{G}(\mathrm{d}, \mathrm{p}) \quad-250.9735(\mathbf{0 . 0 0})$

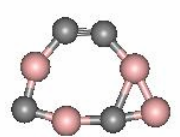

$\left(\mathrm{C}_{s},{ }^{1} \mathrm{~A}^{\prime}\right)$

$-251.6274(\mathbf{1 3 . 0 7})$

$-250.8935(\mathbf{1 2 . 2 3})$

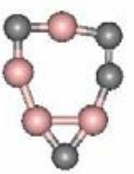

$\left(\mathrm{C}_{s},{ }^{2} \mathrm{~A}^{\prime}\right)$

$-251.7193(-\mathbf{0 . 0 6})$

$-250.9716(\mathbf{1 . 1 8})$

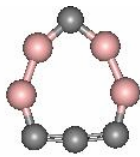

$\left(\mathrm{C}_{2 v},{ }^{1} \mathrm{~A}_{1}\right)$

$-251.6145(\mathbf{2 1 . 1 5})$

$-250.8692(\mathbf{2 7 . 5 0})$

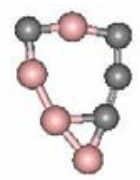

$\left(\mathrm{C}_{s},{ }^{2} \mathrm{~A}^{\prime}\right)$

$-251.7010(11.41)$

$-250.9551(\mathbf{1 1 . 5 3 )}$ 


\section{$\mathrm{C}_{4} \mathrm{~B}_{5}$}

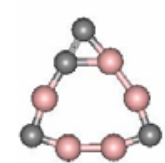

$$
\left(\mathrm{C}_{\mathrm{s}},{ }^{2} \mathrm{~A}^{\prime}\right)
$$

B3LYP/6-311+G(d,p) $\quad-276.4652(\mathbf{0 . 0 0})$

$\operatorname{CCSD}(\mathrm{T}) / 6-311+\mathrm{G}(\mathrm{d}, \mathrm{p}) \quad-275.6460(\mathbf{0 . 0 0})$

$\operatorname{CCSD}(\mathrm{T}) / \mathrm{cc}-\mathrm{pVTZ}$

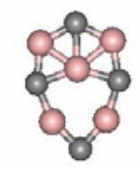

$\left(\mathrm{C}_{s},{ }^{2} \mathrm{~A}^{\prime}\right)$

$-276.4681(-\mathbf{1 . 8 2})$

$-275.6467(-\mathbf{0 . 4 2})$

$-275.7880(\mathbf{2 . 6 4})$

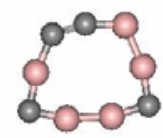

$\left(\mathrm{C}_{s},{ }^{2} \mathrm{~A}^{\prime}\right)$ $-276.4623(\mathbf{1 . 8 2})$

$-275.6346(\mathbf{7 . 2 0})$

\section{$\mathrm{C}_{4} \mathrm{~B}_{5}^{-}$}

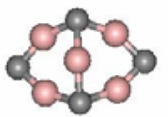

$$
\left(\mathrm{C}_{2 v},{ }^{1} \mathrm{~A}_{1}\right)
$$

B3LYP/6-311+G(d,p)

-276.5658 (0.00)

$\operatorname{CCSD}(\mathrm{T}) / 6-311+\mathrm{G}(\mathrm{d}, \mathrm{p}) \quad-275.7516(\mathbf{0 . 0 0})$

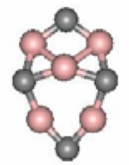

$$
\left(\mathrm{C}_{s},{ }^{1} \mathrm{~A}^{\prime}\right)
$$

-276.5632 (1.62)

$-275.7473(\mathbf{2 . 2 8})$

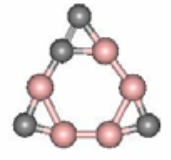

$\left(\mathrm{C}_{s},{ }^{1} \mathrm{~A}^{\prime}\right)$

$-276.5694(\mathbf{2 . 7 1})$

$-275.7412(\mathbf{6 . 5 1})$ 


\section{$\mathrm{C}_{4} \mathrm{~B}_{6}$}

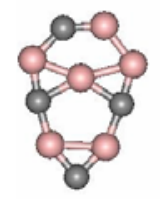

$\left(\mathrm{C}_{\mathrm{s}},{ }^{1} \mathrm{~A}^{\prime}\right)$

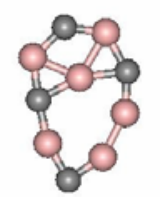

$\left(\mathrm{C}_{s},{ }^{1} \mathrm{~A}^{\prime}\right)$

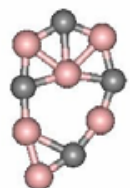

$\left(\mathrm{C}_{1},{ }^{1} \mathrm{~A}\right)$

B3LYP/6-311+G(d,p) $\quad-301.3437(\mathbf{0 . 0 0})$

-301.3426 (0.69)

-301.3218 (13.76)

$\operatorname{CCSD}(\mathrm{T}) / 6-311+\mathrm{G}(\mathrm{d}, \mathrm{p}) \quad-300.4496(\mathbf{0 . 0 0})$

$-300.4462(\mathbf{2 . 1 4})$

-300.4255 (15.14)

\section{$\mathrm{C}_{4} \mathrm{~B}_{6}{ }^{-}$}

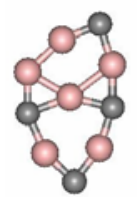

$\left(\mathrm{C}_{s},{ }^{2} \mathrm{~A}^{\prime}\right)$

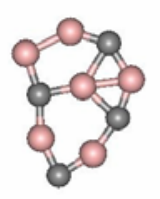

$\left(\mathrm{C}_{\mathrm{s}},{ }^{2} \mathrm{~A}^{\prime}\right)$

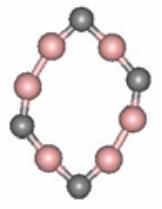

$\left(\mathrm{C}_{2 h},{ }^{2} \mathrm{~A}_{u}\right)$

B3LYP/6-311+G(d,p) $\quad-301.4361(\mathbf{0 . 0 0}) \quad-301.4039(\mathbf{2 0 . 2 0})$

-301.4194 (18.08)

CCSD(T)/6-311+G(d,p) $\quad-300.5330(\mathbf{0 . 0 0}) \quad-300.5042(\mathbf{1 0 . 4 7})$ 


\section{$\mathbf{C}_{4} \mathbf{B}_{7}$}

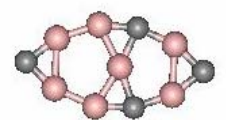

$\left(\mathrm{C}_{2 v},{ }^{2} \mathrm{~A}_{1}\right)$

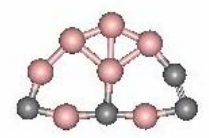

$\left(\mathrm{C}_{s},{ }^{2} \mathrm{~A}^{\prime}\right)$

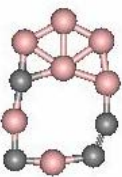

$\left(\mathrm{C}_{s},{ }^{2} \mathrm{~A}\right)$

B3LYP/6-311+G(d,p)

$-326.2008(\mathbf{0 . 0 0})$

-326.1770 (14.93)

-326.1713 (18.52)

CCSD(T)/6-311+G(d,p) $\quad-325.2108(\mathbf{0 . 0 0})$

-325.1803 (19.19)

$-325.1757(\mathbf{2 0 . 0 5})$

\section{$\mathbf{C}_{4} \mathbf{B}_{7}^{-}$}

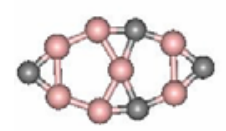

$\left(\mathrm{C}_{2 v},{ }^{1} \mathrm{~A}_{1}\right)$

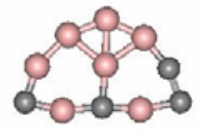

$\left(\mathrm{C}_{s},{ }^{1} \mathrm{~A}^{\prime}\right)$

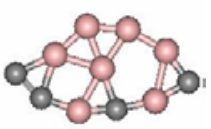

$\left(\mathrm{C}_{\mathrm{s}},{ }^{1} \mathrm{~A}^{\prime}\right)$

B3LYP/6-311+G(d,p) $\quad-326.3243(\mathbf{0 . 0 0}) \quad-326.3041(\mathbf{1 2 . 6 8})$

$-326.2984(\mathbf{1 6 . 2 2})$

$\operatorname{CCSD}(\mathrm{T}) / 6-311+\mathrm{G}(\mathrm{d}, \mathrm{p}) \quad-325.3401(\mathbf{0 . 0 0}) \quad-325.3202(\mathbf{1 2 . 4 8})$

$-325.3182(\mathbf{1 3 . 7 3 )}$ 


\section{$\mathbf{C}_{4} \mathbf{B}_{8}$}

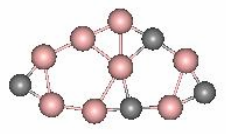

$\left(\mathrm{C}_{\mathrm{s}},{ }^{1} \mathrm{~A}^{\prime}\right)$

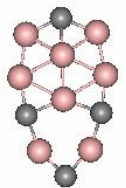

$\left(\mathrm{C}_{1},{ }^{1} \mathrm{~A}\right)$

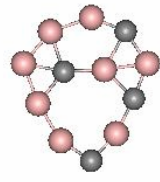

$\left(\mathrm{C}_{\mathrm{s}},{ }^{1} \mathrm{~A}^{\prime}\right)$

B3LYP/6-311+G(d,p) $\quad-351.0570(\mathbf{0 . 0 0}) \quad-351.0388(\mathbf{1 1 . 4 9})$

$-351.0417(9.66)$

$\operatorname{CCSD}(\mathrm{T}) / 6-311+\mathrm{G}(\mathrm{d}, \mathrm{p}) \quad-349.9956(\mathbf{0 . 0 0}) \quad-349.9831(\mathbf{7 . 8 1})$

$-349.9794(\mathbf{1 0 . 1 3})$

\section{$\mathbf{C}_{4} \mathbf{B}_{8}{ }^{-}$}

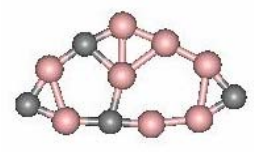

$\left(\mathrm{C}_{s},{ }^{2} \mathrm{~A}\right)$

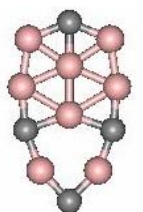

$\left(\mathrm{C}_{2 v},{ }^{2} \mathrm{~A}_{2}\right)$

$-351.1356(\mathbf{1 3 . 2 7})$

$-350.0731(9.51)$

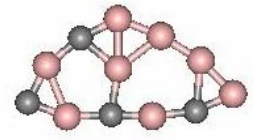

$\left(\mathrm{C}_{s},{ }^{2} \mathrm{~A}^{\prime}\right)$

$-351.1383(\mathbf{1 1 . 6 1 )}$

-350.0703 (11.27) 
III. Geometrical data for structures given in Figure 4.

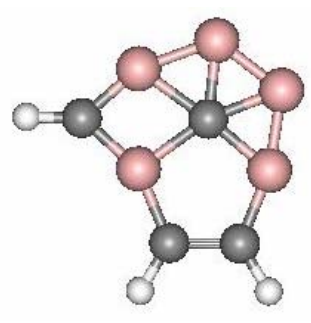

P1- $(\mathrm{CH})_{3}$-iso

Geometry data: (B3LYP/6-311+G(d,p) optimization)

$\begin{array}{llll}\text { C } & -0.379929 & 0.571257 & -0.002131 \\ \text { C } & 2.081266 & 0.132140 & 0.000893 \\ \text { H } & 3.159989 & 0.258749 & 0.002400 \\ \text { C } & 1.506950 & -1.117282 & -0.000749 \\ \text { H } & 2.121735 & -2.014945 & -0.000533 \\ \text { B } & 1.020105 & 1.244169 & 0.000931 \\ \text { B } & -1.821405 & -0.260566 & 0.000665 \\ \text { B } & -1.699841 & 1.350174 & 0.000288 \\ \text { B } & -0.300847 & 2.100152 & 0.000123 \\ \text { C } & -1.399769 & -1.660410 & 0.000549 \\ \text { H } & -1.864423 & -2.631611 & 0.004031 \\ \text { B } & -0.051694 & -1.067213 & -0.001460\end{array}$




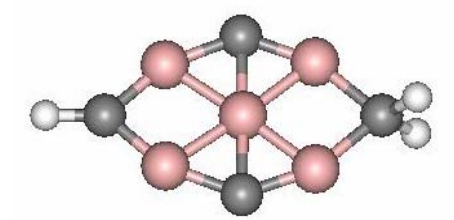

Geometry data: (B3LYP/6-311+G(d,p) optimization)

$\begin{array}{lrrr}\text { B } & 1.282700 & -1.028601 & -0.000130 \\ \text { B } & -1.437232 & -0.971906 & -0.000071 \\ \text { B } & 1.282700 & 1.028601 & -0.000130 \\ \text { B } & -1.437232 & 0.971906 & -0.000071 \\ \text { B } & -0.027096 & 0.000000 & -0.001194 \\ \text { C } & -2.533153 & 0.000000 & 0.000698 \\ \text { C } & -0.056223 & 1.517711 & -0.000366 \\ \text { C } & 2.487436 & 0.000000 & 0.000710 \\ \text { C } & -0.056223 & -1.517711 & -0.000366 \\ \text { H } & -3.610340 & 0.000000 & 0.001611 \\ \text { H } & 3.120682 & 0.000000 & -0.887380 \\ \text { H } & 3.119426 & 0.000000 & 0.889687\end{array}$


IV. Infrared spectrum of the $\mathrm{CB}_{4}$.

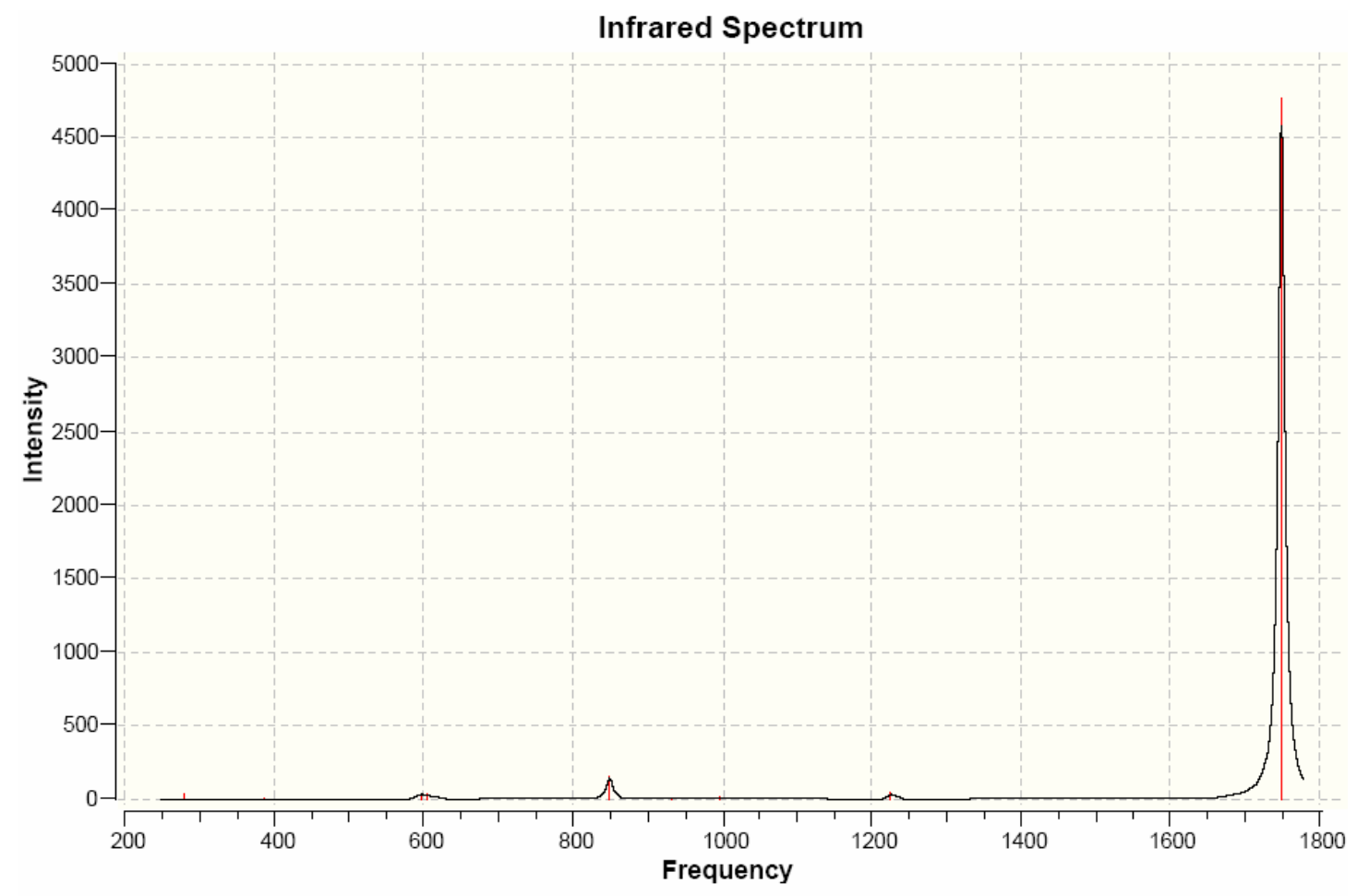

